\title{
Continuous Interescalene Blockade in Postoperative Pain management in Patients Operated of Total shoulder Arthroplasty
}

Acevedo I*., Dominguez $\mathrm{F}^{*}$., Martin $\mathrm{J}^{*}$., Pestaña D.*

*Anestesiology Department. Ramón y Cajal Universitary Hospital. Madrid. Spain.

There is a current trend to use regional techniques for the intraoperative and postoperative pain management, showing a possible benefit with continuous interescalene nerve block (CISNB) compared to single puncture.

The aim of this study is to present our experience in the use of CISNB in the management of postoperative pain in patients undergoing total shoulder arthroplasty.

\begin{abstract}
Background and Goal:
It is well known that the total shoulder arthroplasty (TSA) causes severe pain during the first 24 hours, which could delay the hospital discharge and increase the incidence of adverse effects associated with high doses of opiates. There is a current trend to use regional techniques for the intraoperative and postoperative pain management, showing a possible benefit with continuous interescalene nerve block (CISNB) compared to single puncture.
\end{abstract}

The aim of this study is to present our experience in the use of CISNB in the management of postoperative pain in patients undergoing total shoulder arthroplasty.

\section{Materials and Methods:}

We retrospectively reviewed the records of 7 patients ( 5 female $/ 2$ male), Undergoing TSA under general anesthesia combined with CISNB. The characteristics in terms of age and ASA classification were similar in patients enrolled. All patients were carried to the Postoperative Acute care Unit and discharged between 3 and 5 hours. Interescalene catheter was ultrasound guided placed. In all patients the surgery were done in beach chair position. Before the surgery a bolus of $15 \mathrm{ml}$ of ropivacaine $7,5 \%$ was administered.

In the postoperative period patients were followed by the Acute Pain Management Unit (APMU). Pain assessment by Visual Analogue Scale (VAS), anti-inflammatory medication, numbers of intravenous morphine rescues and side effects were evaluated at 24 and 48 hours.

VAS scale value and PCA morfine requirements. Continuous INB

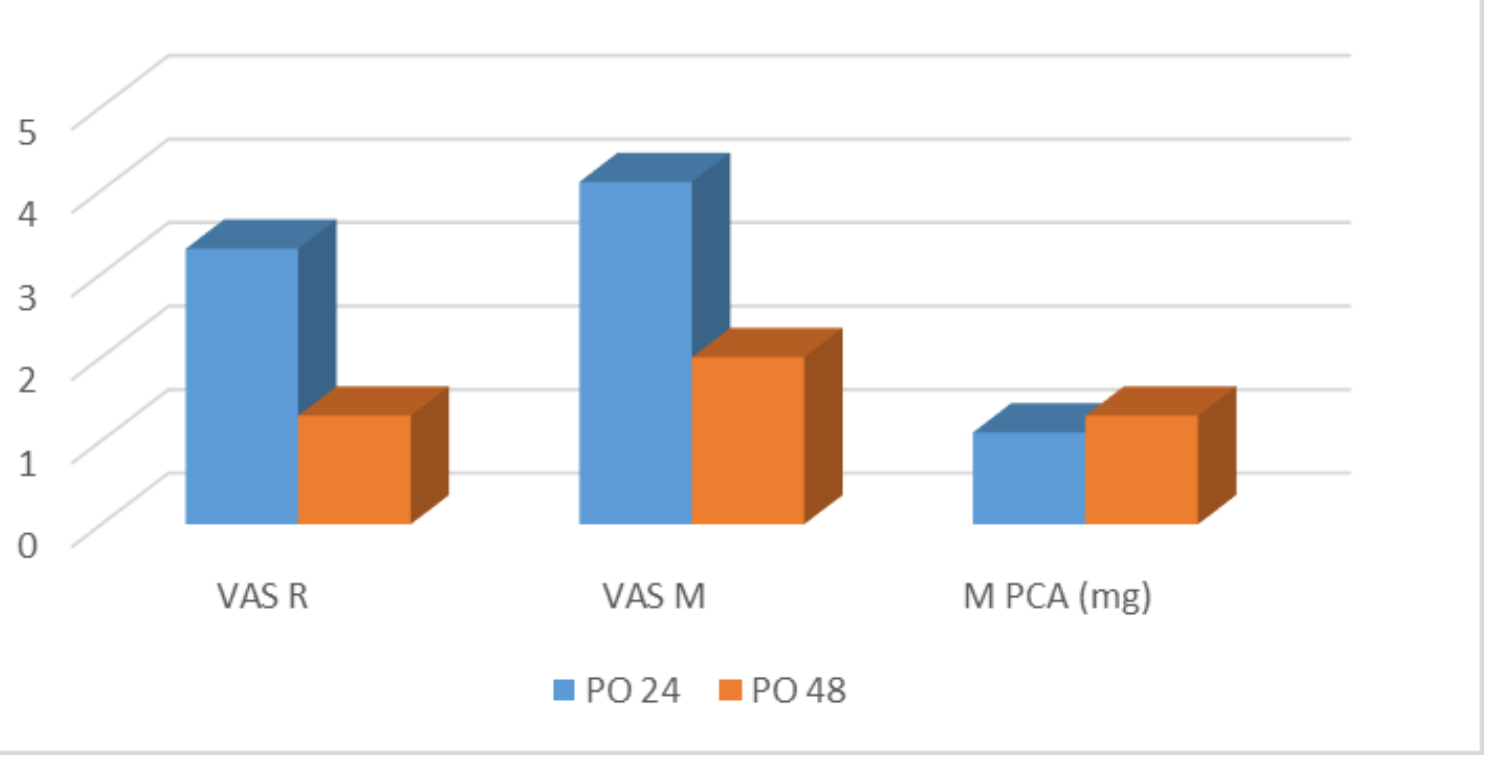

1. Ilfeld BM. Continuous Peripheral Nerve Blocks: An Update of the Published Evidence and Comparison With Novel, Alternative Analgesic Modalities. Anesth Analg. 2017 Jan;124(1):308-335.
Results and Discussion:

Mean age was 81 years (87/65). American Society Anaesthesiologists score was II in 2 patients and III in 5 . In all of them the same pattern of postoperative analgesia was followed using the AMPU intravenous protocols for shoulder surgery (Metamizol $2 \mathrm{~g}$ each 8 hours combined with Paracetamol $1 \mathrm{~g} / 8 \mathrm{~h}$ and ropivacaine 0,2\% trough perineural catheter, programmed with a continuous infussion of $5 \mathrm{ml} / \mathrm{h}$ and bolus of $5 \mathrm{ml}$ each $30 \mathrm{~min}$ ). The VAS scale average at 24 and 48 hours after surgery, at rest and in motion was 3,3 and 4,1 (24 h); 1,3 and 2 (48 h), respectively. Intravenous morphine with PCA requirements average at 24 and 48 was 1,1 mg and 1,3; respectively. No one patient presented side effects.
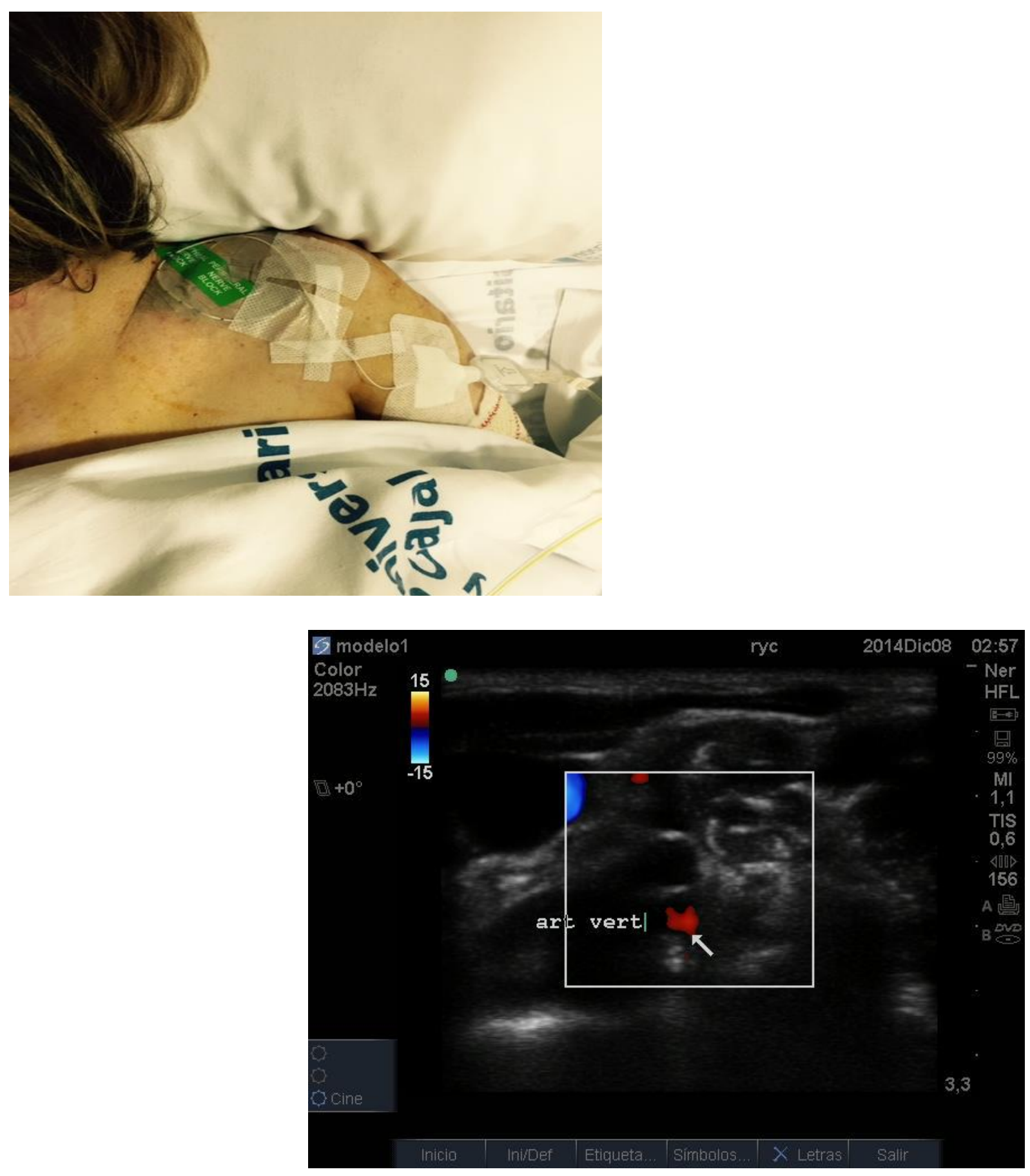

Conclusion

CISNB provides safe and effective postoperative analgesia in the immediate postoperative period for patients with shoulder arthrosis undergoing total shoulder replacement. 